García-Navarrete, P. G., Soria-Ortiz, G. J., \& González-Salazar, C. (2021). Interacciones potenciales parásito-hospedero entre el escarabajo Dendroctonus (Coleoptera: Scolytidae) y Pinus (Pinaceae) en México. Revista de Biología Tropical, 69(3), 1004-1022. https://doi.org/10.15517/rbt. v69i3.45910

\title{
Interacciones potenciales parásito-hospedero entre el escarabajo Dendroctonus (Coleoptera: Scolytidae) y Pinus (Pinaceae) en México
}

\author{
Patricia G. García-Navarrete1; (D) https://orcid.org/0000-0003-1451-7265 \\ Gerardo J. Soria-Ortiz²; (D https://orcid.org/0000-0003-1952-7323 \\ Constantino González-Salazar ${ }^{3,4^{*}}$; (D) http://orcid.org/0000-0001-7347-714X
}

1. Museo de Zoología Alfonso L. Herrera, Departamento de Biología Evolutiva, Facultad de Ciencias, Universidad Nacional Autónoma de México (UNAM), Ciudad de México, México; patricia_gn@live.com.mx

2. Instituto de Ecología, Universidad Nacional Autónoma de México (UNAM), Circuito exterior s/n anexo al Jardín Botánico, Ciudad de México, México; gsoria@ecologia.unam.mx

3. Centro de Ciencias de la Atmósfera, Universidad Nacional Autónoma de México (UNAM), Ciudad de México, México; cgsalazar@atmosfera.unam.mx (*Correspondencia)

4. C3-Centro de Ciencias de la Complejidad, Universidad Nacional Autónoma de México (UNAM), Ciudad de México, México.

Recibido 19-II-2021. Corregido 16-VII-2021. Aceptado 31-VIII-2021.

\section{ABSTRACT \\ Potential parasite-host interactions between Dendroctonus (Coleoptera: Scolytidae) beetle and Pinus (Pinaceae) in Mexico}

Introduction: The parasite-host relationship between bark beetles of the genus Dendroctonus and species of the genus Pinus has been recognized as important threat to coniferous forests because it can cause high tree mortality and therefore a loss of forests.

Objectives: We aimed to identify the potential unknown parasite-host relationships among bark beetles and pine trees species, as well as the contribution of environmental conditions as driver of these ecological interactions. Methods: We carried out ecological niche models and ecological network analysis using the spatial datamining platform SPECIES (http://species.conabio.gob.mx/). Our study included seven species of bark beetles, 52 species of the genus Pinus, and 19 bioclimatic variables from Worldclim dataset.

Results: We identified 140 potential interactions between Dendroctonus and Pinus species, of which $42 \%$ have not been previously registered. Complex inference network analysis allowed us to characterize ecological parasite-host interactions in generalists and specialists. Regarding the ecological niche analysis, we determined potential bark beetle distributions by combining Pinus species ranges and climatic variables as predictors. We noted that climatic variables contributed negatively to Dendroctonus distribution for the most species, i.e. these abiotic variables are restrictive in almost the entire distribution of the beetles; conversely, biotic variables (i.e. Pinus species) were positive and informative on the most of species ranges.

Conclusions: Coexistence patterns of bark beetles and Pinus provide us information on the interactions that are able to establish among these species and that until now can be unknown. In addition, integrating biotic (hosts) and abiotic (climate) factors, allows us to obtain geographic distribution models that characterize the regions with favorable conditions for the presence of bark beetles, as well as the host species with which they could be interacting in those regions. Considering the phytosanitary risk due to bark beetles, our results provide geographic and ecological scenarios where bark beetles infestations may occur, as well as the possible emergence of new parasite-host interactions. These models can be a tool to address future fieldwork efforts to validate and complement our results.

Key words: infestations; data mining; forest health; insects; conifers. 
Los pinos son de gran importancia tanto ecológica como económica, ya que proporcionan diferentes servicios ambientales: captación y filtración de agua, generación de oxígeno, almacén de carbono, retención del suelo y son el hábitat de múltiples especies (Martínez-Méndez et al., 2016). Además, los pinos proporcionan recursos como madera, leña y resinas. Se estiman 111 especies de pinos en el mundo, de las cuales 52 están distribuidas en México y 26 son endémicas (CONABIO, 2021; Sánchez-González, 2008; World Resources Institute, 1995). Los bosques de pino en México se encuentran amenazados por diversas causas antropogénicas como la tala inmoderada, la obtención de productos secundarios y el cambio de uso de suelo para actividades agrícolas (Alvarado et al., 1991). Así mismo, estos pueden verse afectados por agentes biológicos que en algunos casos resultan en plagas, como hongos (ej. Ceratocystiopsis fasciata, Leptographium sp. y Ophistoma pulvinisporum, Moreno-Rico et al., 2015), pulgones (ej. Cinara atlantica y Essigella californica, Hernández-Cruz \& Muñoz-Viveros, 2011; Pietrukiewicz \& Eskiviski, 2010), barrenadores (ej. Eucosma bobana, Retinia arizonensis, Rhyacionia sp., Álvarez-Zagoya et al., 2019; Ruíz-Cancino et al., 1997) y descortezadores (ej. Dendroctonus spp., Billings et al., 2004; del-Val \& Sáenz-Romero, 2017). Estas plagas tienen la capacidad de debilitar y liquidar una gran masa boscosa (Cibrián-Tovar et al., 2007; Cibrián-Tovar et al., 2009; Fairweather et al., 2006; Jiménez-Martínez, 2005).

Desde el punto de vista económico, una de las plagas que ocasiona grandes pérdidas en los bosques de coníferas son los descortezadores del género Dendroctonus (Coleoptera: Scolytidae), los cuales están íntimamente ligados a hospederos como Larix, Picea, Pinus y Pseudotsugae (Bentz et al., 2010; Zúñiga et al., 2002). Se reconocen 20 especies de Dendroctonus, de las cuales 13 se distribuyen en México y parasitan únicamente a especies de Pinus, con los que comparte su historia de especiación, diversificación y dispersión sobre los principales sistemas montañosos de
México (Armendáriz-Toledano et al., 2018; Armendáriz-Toledano \& Zúñiga, 2017; Wood, 1963; Zúñiga et al., 2002).

Debido al riesgo forestal que representa Dendroctonus para los bosques de pino en México, es necesario desarrollar estudios que permitan estimar las preferencias ambientales y ecológicas de los descortezadores e identificar las especies de árboles con el potencial de establecer una interacción con estas especies, así como las regiones con las condiciones ambientales que puedan favorecer la presencia de estas plagas. Entre los principales factores (bióticos y abióticos) que pueden influir en la distribución de los descortezadores se encuentran, sus hospederos, la temperatura y la altitud (Cano et al., 2005; Chapman et al., 2012; Creeden et al., 2014; Hicke et al., 2006; Mendoza et al., 2011b; Morales-Rangel et al. 2016; MoralesRangel et al., 2018; Régnière \& Bentz, 2007; Salinas-Moreno et al., 2004; Soto-Correa et al., 2018; Soto-Correa et al., 2020; Ungerer et al., 1999; Weed et al., 2015). Con esta base, se han desarrollado aproximaciones utilizando sistemas de información geográficas para evaluar las zonas de sobreposición entre descortezadores y pinos, que indirectamente se reconocen como interacciones potenciales de alto riesgo por la sobreposición geográfica de las especies (Salinas-Moreno et al., 2004; Zúñiga et al., 1999).

Adicionalmente se han utilizado modelos de distribución de especies, los cuales relacionan las localidades de presencia de las especies con variables ambientales (Elith et al., 2006), para estimar la distribución potencial de descortezadores en México y Norteamérica (Maldonado et al., 2015; Mendoza et al., 2011a; Salinas-Moreno et al., 2010; Santana-Zarza et al., 2020; Sosa et al., 2018). Incluso algunas aproximaciones han incluido escenarios de cambio climático para estimar la distribución futura de estas especies (Bentz et al., 2010; Evangelista et al., 2011; Méndez-Encina et al., 2020; Morales-Rangel et al., 2018; RiveraRojas et al., 2010). Sin embargo, aunque estas aproximaciones identifican lugares con condiciones climáticas favorables para la presencia 
de descortezadores, no evalúan cuáles especies podrían ser susceptibles de ser infestadas por una o varias especies de descortezadores. Por ello, se deben implementar aproximaciones que puedan inferir las potenciales asociaciones entre descortezadores y pinos, y el ambiente en que se pueden dar estas relaciones.

En la última década se ha desarrollado un método de minería de datos espaciales (Stephens et al., 2009) que permite identificar potenciales asociaciones entre especies mediante redes ecológicas, así como generar mapas de distribución potencial integrado variables bióticas y abióticas (González-Salazar et al., 2013). Este método se ha utilizado para inferir interacciones vector-hospedero para zoonosis (Stephens et al., 2009) y ha sido validado con trabajo de campo identificando nuevos hospederos del patógeno que provoca la Leishmaniasis (Berzunza-Cruz et al., 2015; Stephens et al., 2016). Este marco de análisis espaciales se ha implementado en la plataforma web, http://species.conabio.gob.mx/_Stephens et al., 2019), la cual permite acceder y analizar la información contenida en el Sistema Nacional de Información sobre Biodiversidad de México (SNIB) (CONABIO, 2020). El SNIB-CONABIO contiene información de todas las colecciones nacionales y de proyectos de inventarios bióticos, siendo el principal repositorio de información de flora y fauna para México.

Ante los daños forestales ocasionados por descortezadores en México se debe evaluar la contribución individual y en conjunto de factores bióticos (hospederos) y abióticos (ej. temperatura) en la distribución de descortezadores para la detección oportuna de especies de pinos y áreas con riesgo de presentar brotes potenciales de estas plagas. Por lo cual, este trabajo tuvo como objetivo principal, identificar las asociaciones potenciales entre las especies de Pinus y de Dendroctonus, e identificar áreas potenciales donde puedan ocurrir estas interacciones en México. Para alcanzar estos objetivos se utilizó el método de minería de datos espaciales disponible en la plataforma SPECIES, para obtener redes ecológicas de las potenciales interacciones entre ambas especies, y modelos geográficos con la distribución potencial de los escarabajos combinando factores bióticos y abióticos.

\section{MATERIALES Y MÉTODOS}

Para identificar potenciales interacciones entre las especies de Dendroctonus con las de Pinus, e identificar las áreas geográficas con condiciones favorables para la presencia de descortezadores se realizaron dos modelos: 1) redes ecológicas y 2) modelos de nicho ecológico. Ambos se ejecutaron en la Plataforma de exploración de datos ecológicos SPECIES (http://species.conabio.gob.mx/, Stephens et al., 2019). Esta plataforma realiza un análisis de minería de datos espaciales, implementado un marco bayesiano para identificar asociaciones positivas o negativas basado en el grado de co-ocurrencia entre una clase de interés, $C$, (ej. descortezadores) y una serie de factores potencialmente predictivos, $X$, (ej. pinos) (Stephens et al., 2019).

En SPECIES, para cuantificar el grado de co-ocurrencia, se realiza un muestreo de la presencia de las especies con una rejilla que divide la región de estudio. Seleccionar el tamaño de las celdas que componen la rejilla es una cuestión importante ya que influye directamente en los resultados. A este respecto, existen dos consideraciones principales: 1) el efecto del tamaño de la celda sobre el tamaño efectivo de la muestra estadística a analizar, y 2) su efecto sobre el número de co-ocurrencias; además, debemos considerar la extensión del área de estudio (ej. ecorregión, país, continente). Este tópico ha sido evaluado y discutido previamente (Sierra \& Stephens, 2012; Stephens et al., 2009; Stephens et al., 2019), y en SPECIES se han implementado cuadrículas con tamaños de celda de 8, 16, 32 y $64 \mathrm{~km}^{2}$. En este estudio se utilizaron las celdas de $16 \mathrm{~km}^{2}$. La selección del tamaño de cuadrículas tuvo una motivación estadística buscando maximizar el tamaño de muestra, teniendo 20 celdas en promedio con presencia de las especies objetivo.

Una vez definida la rejilla, se obtiene el número de celdas con presencia de la clase, $C$, 
$\left(n_{i}\right)$, el número de presencias de los predictores, $X,\left(n_{j}\right)$ y el número de coincidencias $\left(n_{i j}\right)$. Con estos datos se identifican las co-ocurrencias significativas calculando las probabilidades condicionales, $P(C \mid X)$, y se aplica el estadístico epsilon, $\varepsilon(C \mid X)$, donde valores de $|\varepsilon|>2$ corresponden a una asociación estadísticamente significativa $(\mathrm{P}<0.05)$. Asignando así, valores positivos (atracción) y negativos (repulsión) a cada par de asociaciones (para más detalles referirse a Stephens et al., 2019). A partir de estas asociaciones se pueden construir modelos de redes ecológicas y de nicho ecológico. Para el cálculo de epsilon se consideró un mínimo de cinco celdas con la presencia de las especies de descortezadores $\left(n_{i}=5\right)$ y de pinos $\left(n_{j}=5\right)$.

Redes ecológicas: Para explorar el riesgo de infección de Pinus por especies parásitas de Dendroctonus, se utilizó el módulo de redes ecológicas de SPECIES (http://species. conabio.gob.mx/comunidad_v0.1.html). Se seleccionaron como nodos fuente a las especies válidas de Dendroctonus y como nodos destino a las de Pinus.

Los nodos fuente fueron siete especies de descortezadores: $D$. adjunctus, $D$. approximatus, D. frontalis, D. jeffreyi, D. mexicanus, $D$. parallelocollis y $D$. valens. En este caso, se consideró a $D$. jeffreyi aun sin cumplir con la configuración inicial de cinco celdas como requisito mínimo con datos de ocurrencia $\left(n_{i}\right.$ $=5$ ), ya que cuenta solo con dos celdas $\left(n_{i}=\right.$ 2). Sin embargo, estos registros se deben propiamente al tamaño de su área de distribución, dado que en México se restringe a la sierra norte de Baja California (Armendáriz-Toledano et al., 2018). En los Nodos destino se seleccionaron las 52 especies de pinos disponibles en la plataforma: $P$. arizonica, $P$. attenuata, $P$. ayacahuite, $P$. caribaea, $P$. cembroides, $P$. contorta, $P$. coulteri, $P$. culminicola, $P$. devoniana, $P$. discolor, $P$. douglasiana, $P$. durangensis, $P$. edulis, $P$. engelmannii, $P$. flexilis, $P$. georginae, $P$. greggii, $P$. halepensis, $P$. hartwegii, $P$. herrerae, $P$. jaliscana, $P$. jeffreyi, $P$. lambertiana, P. lawsonii, P. leiophylla, P. lumhotzii, $P$. luzmariae, $P$. maximartinezii, $P$. maximinoi,
P. monophylla, P. montezumae, $P$. muricata, $P$. nelsonii, $P$. oocarpa, $P$. patula, $P$. pinaster, $P$. pinceana, $P$. ponderosa, $P$. praetermissa, $P$. pringlei, $P$. pseudostrobus, $P$. quadrifolia, $P$. radiata, $P$. remota, $P$. resinosa, $P$. rzedowskii, $P$. strobiformis, $P$. strobus, $P$. sylvestris, $P$. taeda, $P$. tecunumanii y $P$. teocote. En el siguiente enlace se tiene acceso a la plataforma SPECIES con las condiciones iniciales de este estudio: http://species.conabio.gob.mx/comunidad_v0.1.html\#link/?token=fc9b8059df4546 faf29a94fc980bd9e1.

El análisis de redes nos proporciona un listado de pares de asociaciones entre Dendroctonus y Pinus. Para evaluar este listado como modelo predictivo de interacciones se hizo una búsqueda de interacciones conocidas entre descortezadores y pinos. Para evaluar cómo se distribuyen estas interacciones conocidas en nuestro listado, este se ordenó de mayor a menor valor de epsilon y se dividió en deciles. Si el modelo es capaz de identificar interacciones se deberán observar el mayor número de interacciones conocidas en el decil más alto $\left(D_{10}\right)$ y este número debe ir decreciendo hacia los deciles más bajos $\left(D_{l}\right)$. Si este patrón se observa en los resultados, entonces el $D_{10}$ corresponda a las asociaciones candidatas para establecer una interacción ecológica y ser observadas en campo y en $D_{l}$, estarán las asociaciones con menores posibilidades (Stephens et al., 2019).

Se realizó una prueba de $X^{2}$, considerando como hipótesis nula $(\mathrm{Ho})$ que el número de interacciones conocidas se distribuyen homogéneamente en los diez deciles, y como hipótesis alternativa $(\mathrm{Ha})$ que la distribución de las interacciones conocidas entre descortezadores y pinos es heterogénea y que, en general, los mayores porcentajes de interacciones conocidas se presentan en los deciles más altos. También se realizó una segunda prueba de $X^{2}$ para definir si las especies de descortezadores se comportan como generalistas $(\mathrm{Ho})$ o como especialistas $(H a)$ en sus interacciones con los pinos. Ambas pruebas de $X^{2}$ se realizaron utilizando la paquetería vegan 2.5-7 en RStudio 3.4.1 (Oksanen et al., 2020). Finalmente, se 
presentan las asociaciones inidentificadas con un grafo $G$ defino por $(V, E)$, donde $V$ son los nodos (fuente y destino) y las aristas $E$ son las relaciones pareadas entre los nodos determinados por el valor de epsilon.

Nicho ecológico: la plataforma SPECIES permite integrar y explorar la contribución conjunta e individual de variables bióticas (ej. especies) y ambientales (ej. clima, altitud) en la distribución de los taxones y determinar la probabilidad de presencia/no presencia de nuestra especie objetivo en una región geográfica particular (González-Salazar et al., 2013; Stephens et al., 2019). Para generar un modelo de distribución potencial la plataforma calcula una función de puntuación (score):

$$
S(X)=\sum_{i=1}^{n} \ln \frac{P(X i \mid C)}{P(X i \mid \underline{C})}
$$

donde $X$ es el conjunto de variables potencialmente predictoras (bióticas y abióticas) que miden diferentes ejes del nicho ecológico de la especie de interés $C$. Para calcular

$$
P\left(X_{i} \mid C\right)=\frac{N_{c x i}}{N_{c}} \text { y } P\left(X_{i} \mid \underline{C}\right)=\frac{N_{x i}-N_{c x i}}{N_{\underline{c}}}
$$

$N_{c x i}$ es el número de celdas donde la clase $C$ (descortezador) y una variable predictora $X_{i}$ (ej. temperatura) co-ocurren, $N_{x i}$ es el número de celdas con presencia de la variable $X_{i}, N_{c}$ es el número de celdas con presencia de $C$, y $N_{c}$ es el número de celdas sin presencia de $C$. Así, se determina el perfil de nicho $X$ para cada celda de nuestra rejilla, asignando el score correspondiente de cada variable, esto es: $S(X)=S\left(X_{1}\right)+$ $S\left(X_{2}\right)+\ldots+S\left(X_{n}\right)$. Los valores altos/bajos de $S(\boldsymbol{X})$ indican si las condiciones son o no favorables para la presencia de $C$ (para más detalles referirse a Stephens et al., 2019).

Los modelos de nicho se realizaron para las siete especies de Dendroctonus empleadas en el análisis de redes ecológicas. En la sección de "Grupo de variables", es posible elegir como variables predictoras datos taxonómicos de ocurrencia y rasters de valores climáticos. Se seleccionaron las 52 especies de Pinus y las 19 variables climáticas de Worldclim: Bio1 $=$ Temperatura media anual, Bio $2=$ Intervalo diurno promedio, Bio3 = Isotermalidad, Bio4
$=$ Temperatura estacional, Bio5 $=$ Temperatura máxima del mes más cálido, Bio6= Temperatura mínima del mes más frío, Bio7 = Intervalo anual de temperatura, $\operatorname{Bio} 8=$ Temperatura media del trimestre más húmedo, Bio9 = Temperatura media del trimestre más cálido, Bio10 = Temperatura media del trimestre más seco, Bio11 = Temperatura media del trimestre más frío, Bio12 = Precipitación anual, Bio13 = Precipitación del mes más húmedo, Bio14 = Precipitación del mes más seco, Bio15 = Precipitación estacional, Bio16 = Precipitación del trimestre más húmedo, Bio17 = Precipitación del trimestre más cálido, Bio18 = Precipitación del trimestre más seco y Bio19 = Precipitación del trimestre más frío (Hijmans et al., 2005).

La plataforma genera 10 deciles geográficos para visualizar y analizar la contribución (valores de score) de las diferentes variables en la distribución de las especies. Las celdas se ordenan conforme al score en forma decreciente y se selecciona el $10 \%$ de las celdas con valores de score más altos, estas celdas corresponden al decil 10. Después se selecciona el siguiente $10 \%$ de celdas que corresponde al decil 9, y así sucesivamente hasta obtener el decil 1 con el score más bajo. Finalmente se obtiene el valor promedio de score por cada decil. Esto nos permite conocer la importancia relativa de cada tipo de variable de forma individual (biótica y abiótica) y en su conjunto por cada decil. Así mismo, se obtiene un mapa con los valores de score para cada celda, de esta forma se identifican las regiones con condiciones óptimas (score $>0$ ), que favorecen la presencia de la especie, y subóptimas (score $<0)$, que corresponden al anti-nicho, es decir, a los sitios que no presentan condiciones favorables para que esté presente la especie (GonzálezSalazar et al., 2013). Los enlaces de acceso a la plataforma SPECIES con los parámetros para generar los modelos de nicho ecológico para cada especie se encuentran en el Apéndice Digital 1.

Para evaluar la contribución del clima en la distribución de los descortezadores se agruparon las variables bioclimáticas correspondientes a temperatura (Bio 1, Bio 2, Bio 3, Bio 4, 
Bio 5, Bio 6, Bio 7, Bio 8, Bio 9, Bio 10 y Bio 11), y precipitación (Bio 12, Bio 13, Bio 14, Bio 15, Bio 16, Bio 17, Bio 18 y Bio 19), con el fin de observar los scores individuales de estos dos conjuntos de variables e identificar cómo influyen en la distribución de las especies. Para comparar estos dos conjuntos de variables se generó un modelo de regresión lineal simple del promedio de score por decil geográfico en función de los deciles geográficos para cada especie y se obtuvieron las pendientes. Considerando que las condiciones subóptimas para la presencia de una especie corresponden con valores de score < 0, la comparación de las pendientes nos permite identificar que variables (temperatura o precipitación) adquieren valores de score negativos más rápido a través de los deciles y, por lo tanto, mayor número de celdas con condiciones no favorables para la presencia de una especie. Para comparar las pendientes se aplicó la prueba $t$-student, evaluando previamente la igualdad o diferencia de las variancias con la prueba $F$. Las pruebas estadísticas se realizaron con una $\mathrm{P}=0.05$.

\section{RESULTADOS}

Redes ecológicas: Se identificaron 140 pares de asociaciones entre las siete especies de Dendroctonus y 38 de Pinus, con valores de epsilon significativos $(\varepsilon=>2)$ (Apéndice Digital 2). El $58 \%$ de las asociaciones corresponden a interacciones previamente documentadas
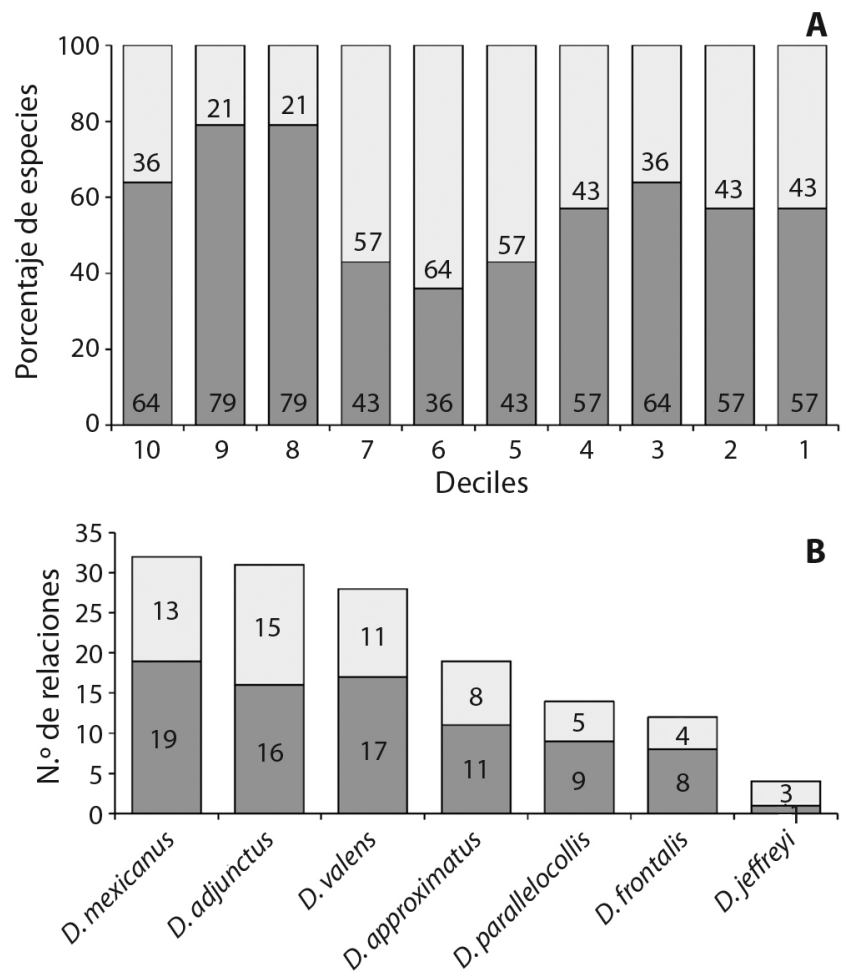

Fig. 1. Relaciones potenciales identificadas en este estudio entre Dendroctonus y Pinus, tanto corroboradas en la literatura (Conocidas, gris oscuro) como no registradas previamente (Desconocidas, gris claro). A. Porcentaje de asociaciones conocidas y desconocidas por decil, del listado de asociaciones obtenido. B. se muestra el número de asociaciones conocidas y desconocidas identificadas por descortezador.

Fig. 1. Potential relationships identified in this study between Dendroctonus and Pinus, both corroborated in the literature (Known, dark gray) and not previously recorded (Unknown, light gray). A. Percentage of known and unknown associations by decile, from the list of associations obtained. B. The number of known and unknown associations identified by bark beetle. 
y el $42 \%$ restante a interacciones sin previo registro en la literatura. $\mathrm{Al}$ evaluar el listado de asociaciones como modelo predictivo se observó que el porcentaje de interacciones conocidas se distribuye heterogéneamente entre los deciles $\left(X i^{2}=50.3, \mathrm{P}<0.05\right.$, g.l. $\left.=9\right)$, donde los porcentajes mayores $(<60 \%)$ se ubican hacia los deciles más altos $\left(D_{10}, D_{9}, D_{8}\right.$; Fig. 1A). Por lo tanto, las asociaciones potenciales observadas en estos deciles son las principales candidatas a ser observadas en campo. Al evaluar la hipótesis que los descortezadores pueden ser generalistas con sus hospederos, observamos que esta no se cumple ya que hay especies especialistas asociándose a pocas especies de pinos $\left(X i^{2}=56.167, \mathrm{P}=0.029\right.$, g.l.
$=38$, Fig. 1B), por lo tanto, estas especies tendrán un impacto restringido a la distribución de sus hospederos.

La red ecológica se compone de 45 nodos (7 descortezadores y 38 pinos) y 140 aristas. Con el fin de facilitar la visualización de las conexiones, se agruparon los nodos según las coincidencias de las relaciones, de esta forma se obtuvieron 17 conjuntos de nodos a los que se les asignó una letra desde la $A$ hasta la $Q$. Por ejemplo, el conjunto $A$ incluye seis especies de pinos que se relacionan cada uno con los mismos seis descortezadores; así mismo, se puede observar la relación de los descortezadores, ej. D. mexicanus (me) que presenta 13 aristas, las

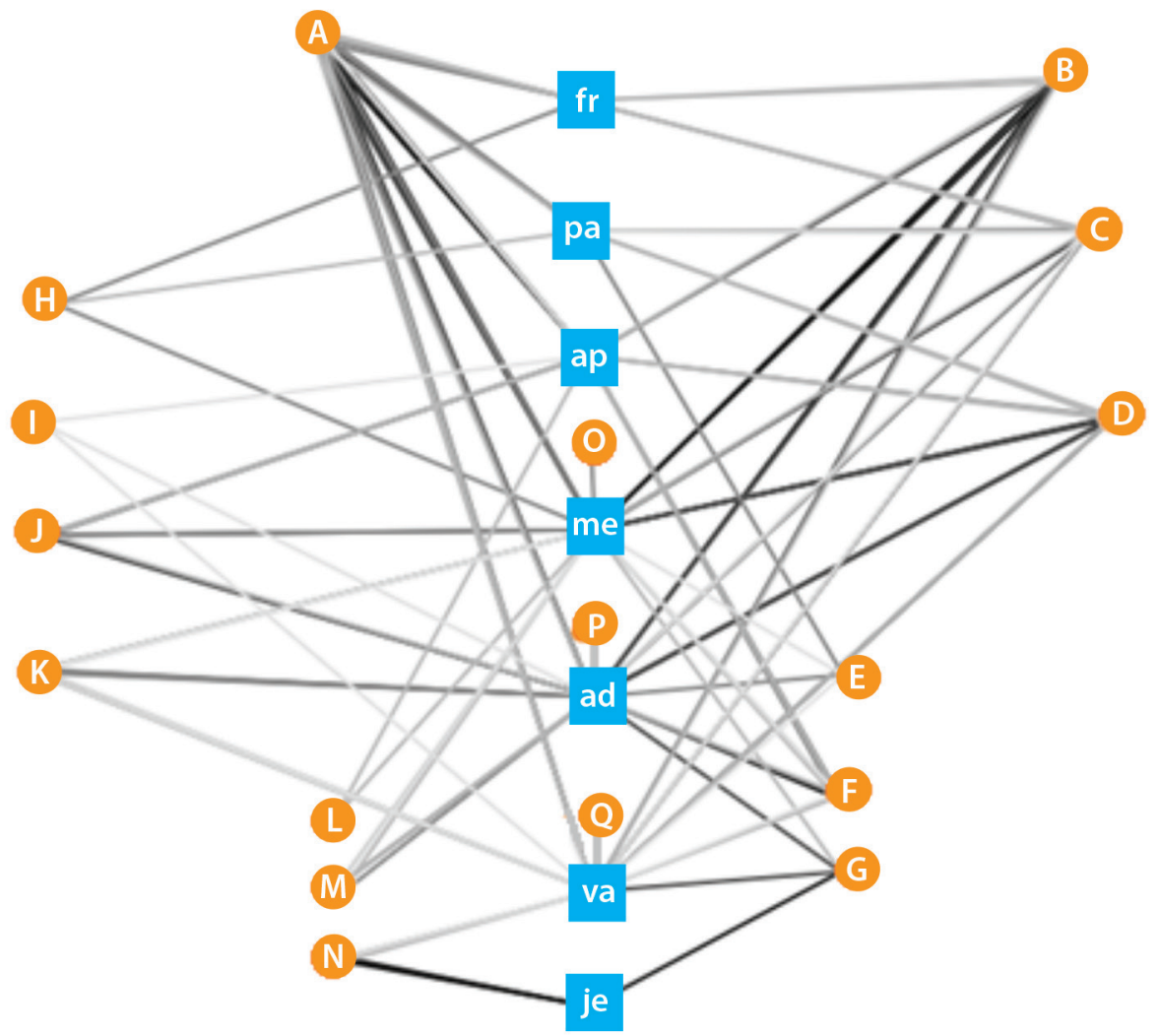

Fig. 2. Red ecológica de Dendroctonus y Pinus. A-Q= Conjuntos de nodos. Nodos: ad= Dendroctonus adjunctus, ap= D. approximatus, $\mathrm{fr}=D$. frontalis, $\mathrm{je}=D$. jeffreyi, $\mathrm{me}=D$. mexicanus, $\mathrm{pa}=D$. parallelocollis $\mathrm{y}$ va $=D$. valens. Las aristas se muestran en escala de grises, las más oscuras corresponden a valores de epsilon más altos.

Fig. 2. Ecological network of Dendroctonus and Pinus. A-Q= Set of nodes. Nodes: $\mathrm{ad}=$ Dendroctonus adjunctus, ap= D. approximatus, $\mathrm{fr}=D$. frontalis, $\mathrm{je}=D$. jeffreyi, $\mathrm{me}=D$. mexicanus, $\mathrm{pa}=D$. parallelocollis and $\mathrm{va}=D$. valens. Edges are shown in grayscale, darker ones correspond to higher epsilon values. 
TABLA 1

Especies de pinos agrupados en los nodos de la red y las asociaciones (*) identificadas con las especies de Dendroctonus y Pinus

TABLE 1

Pine species grouped by nodes of the network and associations (*) identified between Dendroctonus and Pinus species

\begin{tabular}{|c|c|c|c|c|c|c|c|c|c|c|}
\hline $\begin{array}{l}\text { Conjunto } \\
\text { de nodos }\end{array}$ & Especies de Pinus & $a d$ & $a p$ & $f r$ & je & me & $p a$ & va & \#D & $\# \mathrm{P}$ \\
\hline A & $\begin{array}{l}\text { P. hartwegii, P. lawsonii, P. maximinoi, P. montezumae, } \\
\text { P. pringlei y P. pseudostrobus }\end{array}$ & $*$ & $*$ & $*$ & & $*$ & $*$ & $*$ & 6 & 6 \\
\hline $\mathrm{B}$ & P. maximartinezii y P. patula & $*$ & $*$ & $*$ & & $*$ & & $*$ & 5 & 2 \\
\hline $\mathrm{C}$ & P. devoniana, $P$. oocarpa y $P$. teocote & $*$ & & $*$ & & $*$ & $*$ & $*$ & 5 & 3 \\
\hline $\mathrm{D}$ & P. ayacahuite, $P$. douglasiana y P. leiophylla & $*$ & $*$ & & & $*$ & $*$ & $*$ & 5 & 3 \\
\hline $\mathrm{E}$ & P. luzmariae & $*$ & & & & $*$ & $*$ & $*$ & 4 & 1 \\
\hline $\mathrm{F}$ & P. arizonica, $P$. culminicola y $P$. flexilis & $*$ & $*$ & & & $*$ & & $*$ & 4 & 3 \\
\hline G & P. jeffreyi & $*$ & & & $*$ & $*$ & & $*$ & 4 & 1 \\
\hline $\mathrm{H}$ & P. tecunumanii & & & $*$ & & $*$ & $*$ & & 3 & 1 \\
\hline I & P. durangensis & $*$ & $*$ & & & & & $*$ & 3 & 1 \\
\hline $\mathrm{J}$ & P. greggii, $P$. herrerae y $P$. pinceana & $*$ & $*$ & & & $*$ & & & 3 & 3 \\
\hline $\mathrm{K}$ & P. edulis, $P$. nelsonii, $P$. radiata y $P$. remota & $*$ & & & & $*$ & & $*$ & 3 & 4 \\
\hline $\mathrm{L}$ & P. strobus & & $*$ & & & $*$ & & & 2 & 1 \\
\hline M & P. cembroides, $P$. praetermissa y $P$. strobiformis & $*$ & & & & $*$ & & & 2 & 3 \\
\hline $\mathrm{N}$ & P. contorta, $P$. lambertiana y $P$. quadrifolia & & & & $*$ & & & $*$ & 2 & 3 \\
\hline $\mathrm{O}$ & P. halepensis & & & & & $*$ & & & 1 & 1 \\
\hline $\mathrm{P}$ & P. engelmannii & $*$ & & & & & & & 1 & 1 \\
\hline \multirow[t]{2}{*}{ Q } & P. ponderosa & & & & & & & $*$ & 1 & 1 \\
\hline & Total & 12 & 7 & 4 & 2 & 13 & 5 & 11 & & \\
\hline
\end{tabular}

Nodos: $\mathbf{a d}=$ Dendroctonus adjunctus, $\mathbf{a p}=D$. approximatus, $\mathbf{f r}=D$. frontalis, $\mathbf{j e}=D$. jeffreyi, $\mathbf{m e}=D$. mexicanus, $\mathbf{p a}=$ D. parallelocollis y va $=$ D. valens. Número de especies de cada conjunto de nodos de Dendroctonus (\#D) y Pinus (\#P).

Nodes: $\mathbf{a d}=$ Dendroctonus adjunctus, $\mathbf{a p}=D$. approximatus, $\mathbf{f r}=D$. frontalis, $\mathbf{j e}=D$. jeffreyi, $\mathbf{m e}=D$. mexicanus, $\mathbf{p a}=D$. parallelocollis and va $=$ D. valens. Number of species from each node set of Dendroctonus (\#D) and Pinus (\#P).

cuales corresponden con 32 especies de pinos (Fig. 2, Tabla 1).

Nicho ecológico: Los resultados indican que para Dendroctonus el Grupo de variables taxonómicas Pinus presenta score positivo en la mayoría de los deciles, mientras que el Grupo de factores climáticos presenta valores negativos desde los primeros deciles en todas las especies (Fig. 3). Los sitios con las condiciones óptimas (celda con score $>0$ ) para $D$. adjunctus, D. approximatus, $D$. mexicanus y $D$. valens se ubican en el centro de México sobre los principales sistemas montañosos; para $D$. frontalis y $D$. parallelocollis, sobre las montañas del sur de México y para D. jeffreyi, al norte de México y sobre la península de Baja
California (Fig. 4A, Fig. 4B, Fig. 4C, Fig. 4D, Fig. 4E, Fig. 4F, Fig. 4G).

Los modelos de regresión de D. adjunctus, D. approximatus y $D$. valens muestran las pendientes más negativas para las variables de temperatura (Fig. 5, Tabla 2). Esta variable adquiere valores de score negativos más rápido a través de los deciles. Para estas especies la temperatura sería el factor que más limita su distribución, ya que una mayor área tendría condiciones subóptimas (score $<0$ ). Para $D$. frontalis y $D$. parallelocollis la pendiente más negativa se observó en la precipitación (Fig. 5 ), sin embargo, no se observaron diferencias significativas con la temperatura (Tabla 2). Para $D$. mexicanus el resultado fue similar, no se observaron diferencias significativas entre 

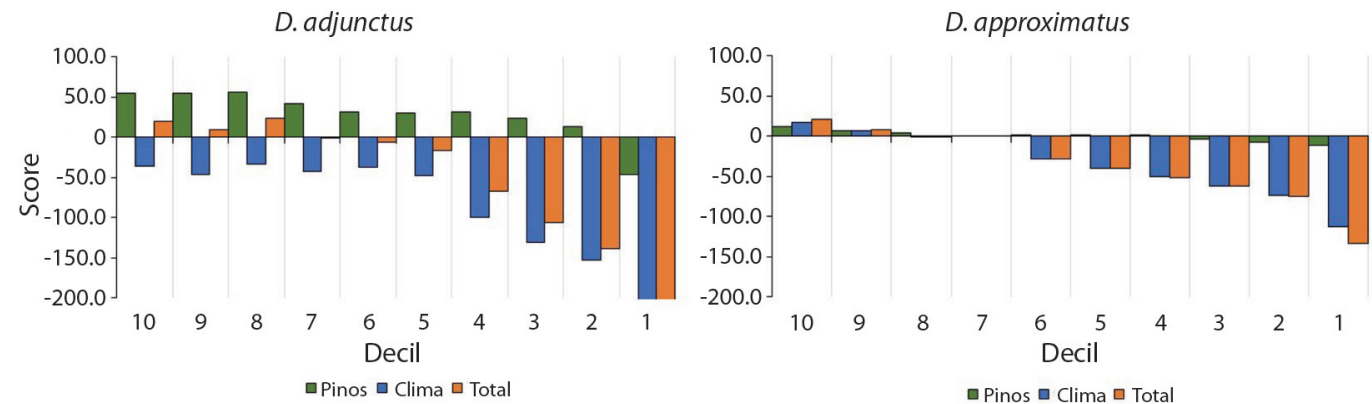

D. frontalis
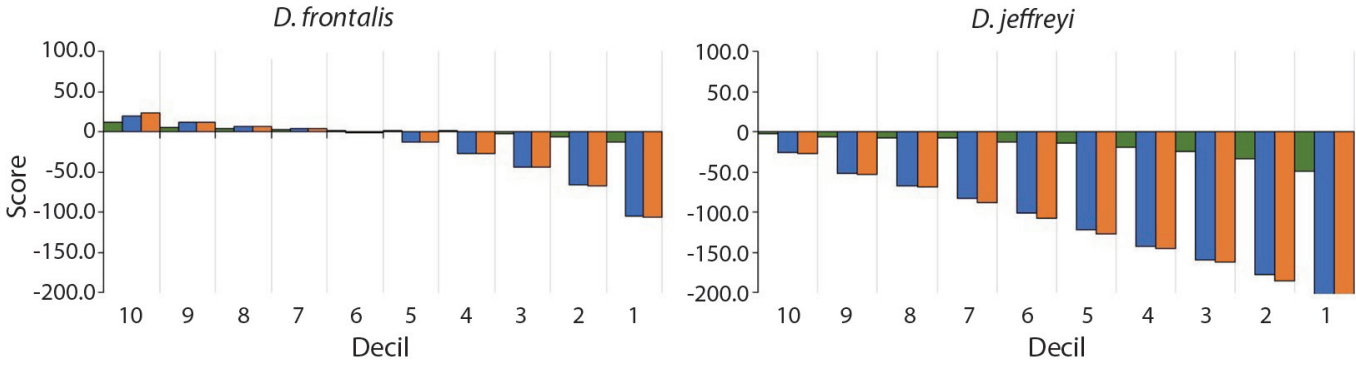

घinos $\square$ Clima $\square$ Total

$\square$ Pinos $\square$ Clima $\square$ Total

D. mexicanus

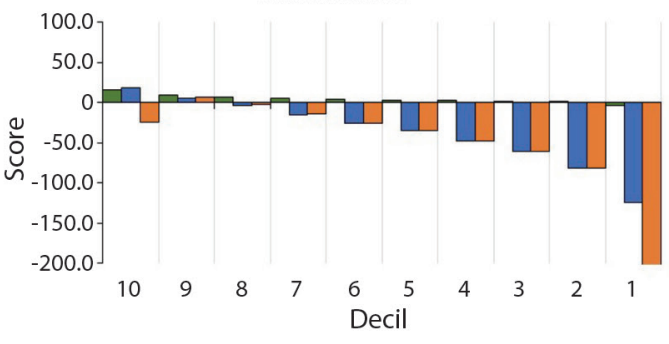

aPinos घ Clima $\square$ Total

D. valens

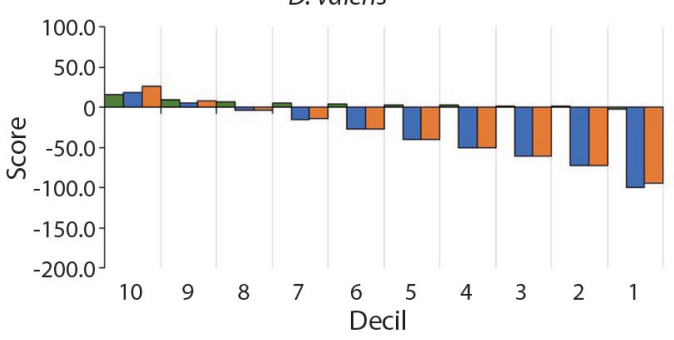

घPinos $\square$ Clima $\square$ Total
D. parallelocollis

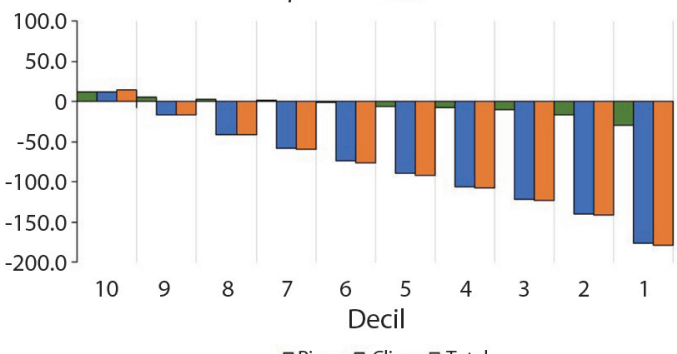

uPinos घ Clima a Total

Fig. 3. Valores de score por decil para el grupo de variables taxonómicas (verde), climáticas (azul) y el score total de las variables en conjunto (naranja) para cada especie.

Fig. 3. Score values per decile of each group of taxonomic (green) and climatic (blue) variables, and the total score of all grouped variables (orange) by each specie. 


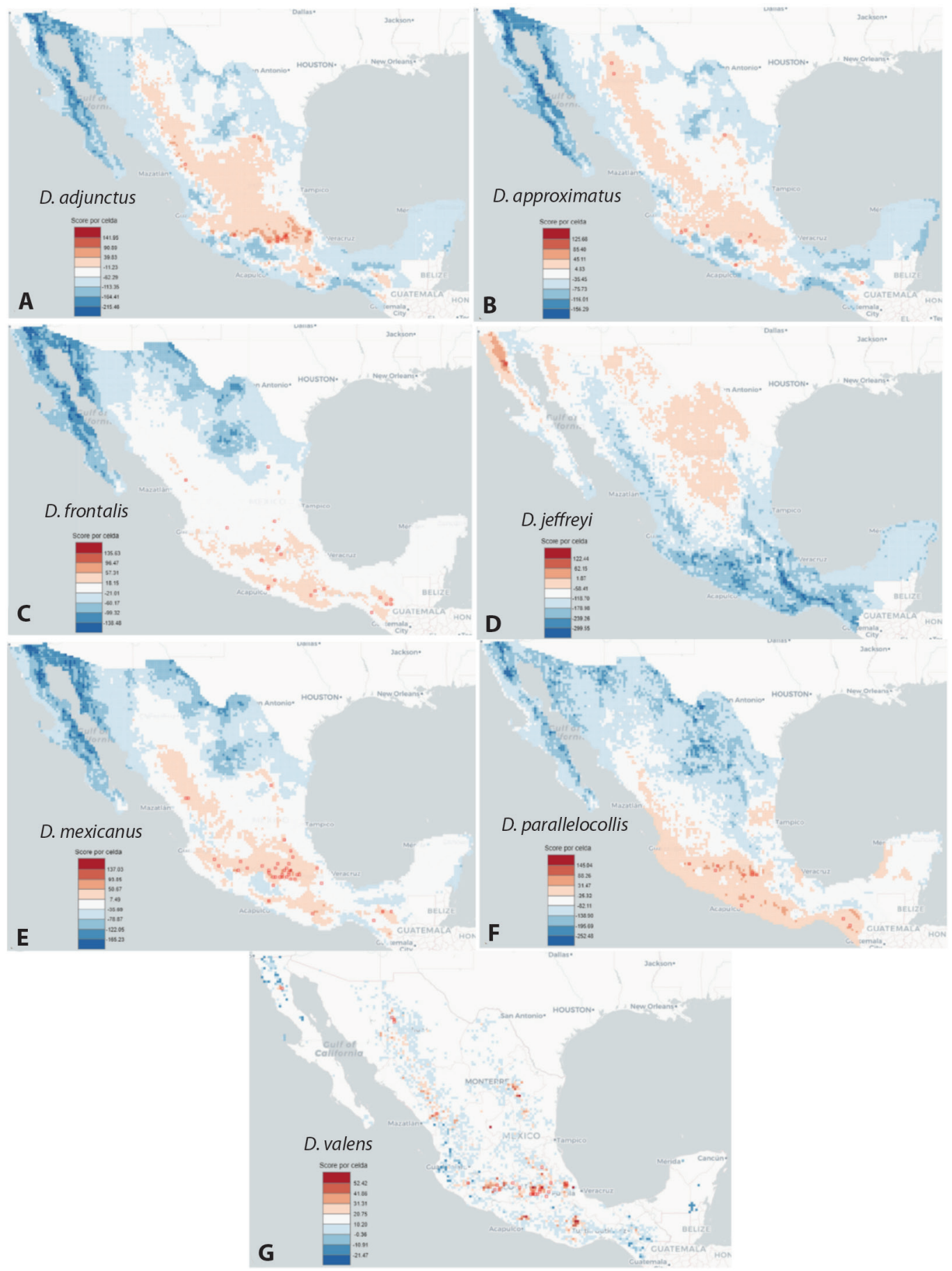

Fig. 4. Modelos de distribución potencial para las especies de Dendroctonus. $\mathrm{A}=D$. adjunctus, $\mathrm{B}=$ D. approximatus, $\mathrm{C}=$ D. frontalis, $\mathrm{D}=D$. jeffreyi, $\mathrm{E}=D$. mexicanus, $\mathrm{F}=D$. parallelocollis y $\mathrm{G}=D$. valens.

Fig. 4. Potential distribution models by Dendroctonus species. $\mathrm{A}=$ D. adjunctus, $\mathrm{B}=$ D. approximatus, $\mathrm{C}=$ D. frontalis, $\mathrm{D}$ $=D$. jeffreyi, $\mathrm{E}=D$. mexicanus, $\mathrm{F}=D$. parallelocollis and $\mathrm{G}=D$. valens . 

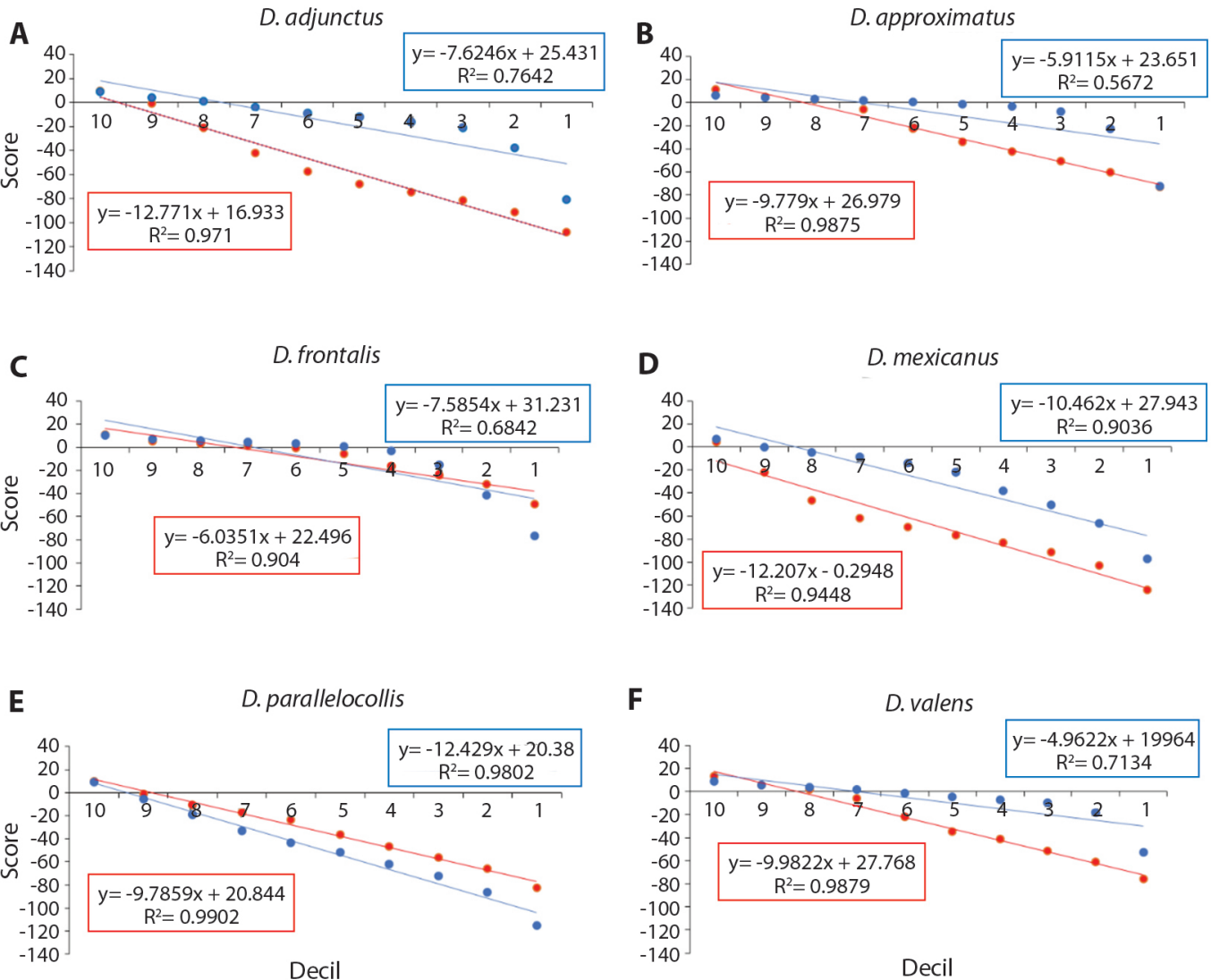

- Tm • Pp

$\bullet \mathrm{Tm} \bullet \mathrm{Pp}$

Fig. 5. Dispersión de los valores de score (eje-y) en función de los deciles (eje-x) para la temperatura (Tm) y precipitación $(\mathrm{Pp})$ por especie: $\mathrm{A}=D$. adjunctus, $\mathrm{B}=D$. approximatus, $\mathrm{C}=D$. frontalis, $\mathrm{D}=D$. mexicanus, $\mathrm{E}=D$. parallelocollis $\mathrm{y} \mathrm{F}=$ D. valens. Se presenta la pendiente obtenida para las variables agrupadas de temperatura (rojo) y precipitación (azul) con el modelo de regresión lineal simple.

Fig. 5. Dispersion graphs of score values (y-axis) as a function of deciles (x-axis) of temperature (Tm) and precipitation $(\mathrm{Pp})$ by species: $\mathrm{A}=D$. adjunctus, $\mathrm{B}=D$. approximatus, $\mathrm{C}=D$. frontalis, $\mathrm{D}=D$. mexicanus, $\mathrm{E}=D$. parallelocollis and $\mathrm{F}$ $=D$. valens. The slope of temperature (red) and precipitation (blue) variables obtained by simple linear regression model is included within the graphs.

temperatura y precipitación (Tabla 2). Para estas especies la combinación de condiciones no favorables de ambos factores estaría limitando su distribución. Este análisis no se realizó para $D$. jeffreyi por la falta de datos.

\section{DISCUSIÓN}

Redes ecológicas: El marco de análisis implementado en este trabajo permitió identificar potenciales interacciones que se pueden establecer entre descortezadores y pinos. De los 140 pares de asociaciones encontradas, observamos que $58 \%$ se han confirmado previamente en campo (Armendáriz-Toledano et al., 2018; Salinas-Moreno et al., 2004). Este resultado nos indica que el método utilizado fue capaz de identificar interacciones conocidas entre las especies de estudio y, por lo tanto, el $42 \%$ de interacciones potenciales tendrían una alta probabilidad de estar presente en la naturaleza por la coexistencia de Dendroctonus y Pinus en el mismo espacio geográfico y 
TABLA 2

Valores de las pendientes del conjunto de variables de temperatura ( $\mathrm{Tm})$ y precipitación (Pp) para las especies de Dendroctonus y de los estadísticos $F$ y $t$

TABLE 2

Slope values of the set of temperature $(\mathrm{Tm})$ and precipitation $(\mathrm{Pp})$ variables of Dendroctonus species and $F$ and $t$ statistics

\begin{tabular}{lcccccc}
\hline \multicolumn{1}{c}{ Especie } & $\mathrm{Tm}$ & $\mathrm{Pp}$ & Valor $F$ & $P$-valor & Valor $t$ & $P$-valor \\
\hline D. adjunctus & -12.771 & -7.763 & 2.34 & 0.13 & 3.67 & $0.001^{*}$ \\
D. approximatus & -9.779 & -5.9115 & 4.70 & 0.02 & 2.31 & $0.016^{*}$ \\
D. frontalis & -6.035 & -7.585 & 2.62 & 0.10 & -0.98 & 0.829 \\
D. mexicanus & -12.207 & -10.462 & 1.16 & 0.42 & 1.16 & 0.130 \\
D. parallelocollis & -9.786 & -12.429 & 1.82 & 0.21 & -2.69 & 0.992 \\
D. valens & -9.982 & -4.962 & 2.85 & 0.08 & 4.10 & $0.0003^{*}$ \\
\hline
\end{tabular}

$\mathrm{El} *$ indica valores estadísticamente significativos

The * indicates statistically significant values.

ecológico, lo que podría repercutir en daños por infestaciones en el bosque de pinos en México que no han sido considerados en el monitoreo de estas plagas.

De las 59 asociaciones potenciales identificadas, nueve fueron previamente propuestas como posibles por Kelley y Farrel (1998), quienes realizaron un estudio de la amplitud de dieta para identificar aquellas especies generalistas que potencialmente pueden parasitar pinos con características similares a sus hospederos conocidos. Este resultado muestra el potencial que hay en el análisis de las bases de datos geográficos, que nos permiten inferir relaciones ecológicas que requerirían un gran esfuerzo humano y alto coste económico para realizar observaciones directas en campo y con una cobertura geográfica regional.

Los descortezadores se relacionaron con el $73 \%$ (38 especies) de los pinos disponibles en el repositorio de datos de la plataforma SPECIES. Esta cifra podría variar según el número de especies que se reconozcan para cada autor. Sánchez-González (2008) realizó un compendio de las especies de Pinus que se han propuesto, las cuales oscilan entre 17 y 55 ; en ese mismo estudio, considerando datos moleculares de distintos autores (Gernandt et al., 2005; Liston et al., 1999; Ramírez-Herrera et al., 2005), se reconocieron 46 especies de Pinus, de las cuales solo $P$. johannis está ausente en la base de datos de SPECIES y por el contrario, del total de especies de pinos de este repositorio, siete no se reconocen en la clasificación de Sánchez-González (2008): P. georginae, $P$. halepensis, $P$. luzmariae, $P$. pinaster, $P$. resinosa, $P$. sylvestris y $P$. taeda. De tal forma que el registro de hospederos para Dendroctonus es dependiente de la clasificación que se tome como base y de los cambios taxonómicos que se ajusten en la plataforma. Sin embargo, las especies del repositorio de la plataforma, que provienen de los datos del SNIB-CONABIO (2020), son consideradas especies validadas para México por autoridades taxonómicas y por lo tanto son adecuados para este tipo de estudios considerando la complejidad taxonómica de los pinos.

La problemática con la clasificación y el reconocimiento de especies válidas de Pinus radica en la similitud entre las especies, lo cual lleva a identificarlas erróneamente; por ejemplo $P$. lawsonii, para la que se han registrado tres especies similares con las que se podría confundir en México: $P$. montezumae, $P$. oocarpa y $P$. teocote (Naturalista, 2020); estos tres pinos se han indicado como hospederos para al menos cinco descortezadores, mientras que $P$. lawsonii se ha registrado como hospedero de $D$. frontalis y $D$. mexicanus (ArmendárizToledano et al., 2018; Salinas-Moreno et al., 2004) Sin embargo, en este trabajo se encontró 
que $P$. lawsonii se podría relacionar con $D$. adjunctus, D. approximatus, D. parallelocollis y D. valens.

Adicionalmente, cabe señalar las relaciones que se han registrado en la literatura (Armendáriz-Toledano et al., 2018; Kelley \& Farrell, 1998; Salinas-Moreno et al., 2004), pero que no se obtuvieron en este análisis (ej. $D$. adjunctus - P. ponderosa, D. approximatus - P. devoniana, D. frontalis - P. arizonica, D. mexicanus - $P$. durangensis y D. valens $-P$. cembroides); puede deberse a que las especies de pinos no cuentan con la cantidad de registros que se consideraron en este trabajo para ser incluidos $\left(n_{i}=5\right)$.

Las asociaciones potenciales son susceptibles de ser verdaderas si además de la coexistencia geográfica se comparte afinidad filogenética, esto con base en la premisa de que las especies emparentadas filogenéticamente comparten características similares, así como una historia evolutiva y de diversificación, la cual está íntimamente ligada con sus hospederos (Bentz et al., 2010; Zúñiga et al., 1999). Por lo tanto, las especies cercanas o que pertenecen a clados o grupos similares podrían coincidir también en sus hospederos. Se han propuesto agrupaciones artificiales para las especies de Dendroctonus con base en las similitudes biológicas, morfológicas, etológicas, ecológicas y de cromosomas (Wood, 1963; Zúñiga et al., 2002). Dichas agrupaciones se han corroborado como naturales de acuerdo con análisis filogenéticos empleando datos de ADN (Kelley \& Farrell, 1998; Víctor \& Zúñiga, 2016). Armendáriz-Toledano et al. (2018) y Six y Bracewell (2015) resumen las características para cada grupo filogenético de las especies de Dendroctonus con respecto su distribución, el cariotipo, la presencia o ausencia de dimorfismo sexual por tamaño, las especies de hospederos, la ubicación de la colonización en el tronco de los hospederos, el tipo de alimentación de la larva, la estrategia del ciclo de vida y su distribución. Las especies de descortezadores empleadas en este estudio se ubican en tres grupos: 1) $D$. parallelocollis, D. terebrans, D. rhizophagus y $D$. valens, 2) D. jeffreyi y D. ponderosae; y 3)
D. adjunctus, D. approximatus, D. brevicomis, $D$. mexicanus, $D$. vitei, $D$. frontalis y $D$. mesoamericanus. Los resultados de este estudio son limitados y no permiten comprobar la hipótesis de que los hospederos se comparten entre los descortezadores del mismo grupo, por lo que esta cuestión habría de abordarse a fondo en estudios más especializados que logren realizar un análisis más completo.

En cuanto al valor de epsilon, se puede observar que su valor más alto (21.10) se presenta para $D$. jeffreyi - P. contorta, ambas especies con área de distribución restringida sobre la sierra de Baja California en México (Armendáriz-Toledano et al., 2018; Gymnosperm Database, 2020), por lo que, a pesar de ser un registro que no se ha propuesto en la literatura resulta factible esta relación dada su coincidencia geográfica. Por otra parte, el valor más bajo (2.01) se presenta entre $D$. valens - $P$. quadrifolia, la cual sí se ha registrado como conocida (Armendáriz-Toledano et al., 2018; Salinas-Moreno et al., 2004). En conclusión, el análisis del listado de asociaciones (descortezador-pino) nos brinda un modelo predictivo de interacciones ecológicas susceptibles de observarse en la naturaleza. Este listado junto con los modelos geográficos son herramientas para dirigir esfuerzos de muestreo en las regiones que reúnen las condiciones para que las especies coexistan. Esto permitirá, por un lado, corroborar las asociaciones propuestas como posibles en este estudio y, segundo, prevenir un posible brote de infestación por descortezadores.

Nicho ecológico: Los resultados de los modelos de nicho mostraron que la distribución de las especies de Dendroctonus está determinada principalmente por sus hospederos (i.e. score $>0$ en la mayoría de los deciles geográficos), lo cual, es un resultado esperado debido a que son especies parasitas que dependen de sus hospederos para completar sus ciclos de vida. Sin embargo, este resultado destaca la necesidad de integrar la presencia de los hospederos cuando se tiene como objetivo identificar áreas geográficas con las condiciones para la 
presencia de descortezadores. Previos estudios han utilizado principalmente modelos basados en el nicho climático para inferir la distribución potencial de los descortezadores y por lo tanto desestiman el papel de los pinos como factores del nicho de estas especies (ej., Maldonado et al., 2015; Mendoza et al., 2011a; Santana-Zarza et al., 2020; Sosa et al., 2018).

Además, debemos destacar que las variables de temperatura y precipitación mostraron valores de score negativos en la mayoría de los deciles geográficos (i.e. regiones con condiciones subóptimas), lo cual, indicaría distribuciones restringidas para la mayoría de las especies por condiciones climáticas. Sin embargo, hay especies con una distribución amplia encontrándose en todos los sistemas montañosos de México (ej., D. approximatus). Aunque estos resultados pueden parecer contradictorios con lo observado previamente, donde se ha destacado la temperatura como una variable que puede favorecer el desarrollo y la distribución de ciertas especies, por ejemplo: D. adjunctus (Cano et al., 2005), D. frontalis (Morales-Rangel et al., 2016; Soto-Correa et al., 2018), D. mexicanus (Soto-Correa et al., 2020), D. ponderosae (Creeden et al., 2014; Hicke et al., 2006) y D. rhizophagus (Mendoza et al., 2011b), y para el género (Armendáriz-Toledano et al., 2018; Salinas-Moreno et al., 2010). Hay que considerar que estos estudios se han enfocado en estudios locales que caracterizan el microambiente que proporcionan los pinos, y al parecer estas condiciones no están siendo capturadas por las variables macro-climáticas utilizadas en este estudio. Debido a que estudios previos han generado modelos de distribución utilizando únicamente el mismo conjunto de variables macro-climáticas, abre la posibilidad de una subestimación de las áreas potenciales para la presencia de descortezadores. Por lo tanto, al realizar modelos de distribución potencial para las especies de Dendroctonus, se debe considerar, además del clima, la distribución simpátrica con sus hospederos y así considerar el microambiente que estos les proporcionan.

Los modelos de distribución potencial para los descortezadores muestran que en general las zonas con mayor score se circunscriben a los sistemas montañosos de México, tal como se ha mencionado por algunos autores (Armendáriz-Toledano et al., 2018; Salinas-Moreno et al., 2004; Sosa et al., 2018). Los mapas de $D$. frontalis, $D$. mexicanus y $D$. valens son los que más se asemejan a la propuesta de distribución geográfica potencial de Armendáriz-Toledano et al. (2018). Sin embargo, los mapas de $D$. adjunctus, D. approximatus, D. jeffreyi y $D$. parallelocollis presentan áreas idóneas fuera de los sistemas montañosos. Esto abre la posibilidad que estas especies puedan tener una distribución mayor a la conocida actualmente. $\mathrm{La}$ especie que mejor ilustra esta situación es $D$. jeffreyi, para la cual los modelos potenciales de Armendáriz-Toledano et al. (2018) la delimitan al norte de Baja California, y en este estudio se presentan áreas favorables (score positivo), en otras regiones del norte del país. Ciertamente, esto son modelos teóricos y no necesariamente implica que las especies estén presentes en las regiones predichas, sin embargo, brindan una herramienta para dirigir esfuerzos de monitoreo en regiones donde no se ha considerado la presencia de un descortezador en particular.

$\mathrm{Al}$ identificar las regiones de mayor score, se facilita la detección de sitios de coexistencia de varias especies de descortezadores que también resulta de suma importancia ya que representan sitios de mayor riesgo potencial de infestación de pinos. En este estudio, la Faja Volcánica Transmexicana es la región en la cual se distribuyen todas las especies de descortezadores, excepto $D$. jeffreyi, por lo tanto, la presión sobre los pinos será mayor en esta región. Zúñiga et al. (1999) indican que los sitios de mayor coexistencia son la Sierra Madre Occidental y la Faja Volcánica Mexicana, mientras que para Salinas-Moreno et al. (2004) la Sierra Madre Occidental y el sur de la Sierra Madre Oriental son las zonas con el mayor número de descortezadores.

Respecto a los análisis de temperatura y precipitación, se destacan dos resultados principales, para $D$. adjunctus, D. approximatus y D. valens la temperatura es el principal factor limitante en su distribución, al presentarse una región mayor con condiciones subóptimas (score $<0)$ de esta variable en comparación con 
la precipitación. Por el contrario, para $D$. frontalis y $D$. mexicanus y $D$. parallelocollis, tanto la temperatura como la precipitación restringen su distribución al combinarse condiciones no favorables de estos factores en la geografía. Contrario a estos resultados Morales-Rangel et al. (2016) no encontraron ninguna correlación directa entre la abundancia de $D$. frontalis y $D$. mexicanus con la humedad relativa mínima, sin embargo, Soto-Correa et al. (2020) concluyeron que la abundancia de $D$. mexicanus si está limitada tanto por temperatura como por la baja humedad.

El hecho que la temperatura represente una variable importante en la distribución de los descortezadores da soporte a los estudios realizados sobre las variaciones que tendrían las poblaciones de descortezadores con aumentos de temperatura en escenarios de cambio climático (Bentz et al., 2010; Evangelista et al., 2011; Méndez-Encina et al., 2020; MoralesRangel et al., 2018; Rivera-Rojas et al., 2010). Sin embargo, debemos considerar la relevancia de los hospederos en la distribución de estas especies, tal como se describió en este estudio, ya que son parte principal de su ciclo de vida e incluso se han señalado hospederos preferentes para cada especie de descortezador (Armendáriz-Toledano et al., 2018; Salinas-Moreno et al., 2004; Salinas-Moreno et al., 2010). Por lo tanto, futuras aproximaciones para estudios de cambio climático deben integrar el papel de los hospederos en las proyecciones futuras para estas especies.

Debido a los impactos de las infestaciones por Dendroctonus en los bosques de pino, resulta indispensable anticiparse a la aparición de estas plagas para lograr aplicar oportunamente medidas de prevención del daño en el recurso forestal. En este sentido, en algunos estudios se ha indicado que $D$. adjunctus, $D$. frontalis y $D$. mexicanus presentan un comportamiento agresivo de colonización (Armendáriz-Toledano et al., 2018), y merecen atención especial, dado el posible riesgo forestal que representan. Por lo cual, se sugiere enfatizar sistemas de vigilancia en los hospederos potenciales de estos descortezadores. Finalmente, los resultados de este estudio junto con futuro trabajo de campo in situ suman a los esfuerzos por mitigar y prevenir el daño causado por los descortezadores en los bosques de pino en México.

Declaración de ética: los autores declaran que todos están de acuerdo con esta publicación y que han hecho aportes que justifican su autoría; que no hay conflicto de interés de ningún tipo; y que han cumplido con todos los requisitos y procedimientos éticos y legales pertinentes. Todas las fuentes de financiamiento se detallan plena y claramente en la sección de agradecimientos. El respectivo documento legal firmado se encuentra en los archivos de la revista.

Ver Apéndice digital en: revistas.ucr.ac.cr

\section{AGRADECIMIENTOS}

Agradecemos a Angela Cuervo-Robayo y Enrique Martínez Meyer por sus comentarios y sugerencias que permitieron mejorar este manuscrito. Los dos primeros autores agradecen al Posgrado en Ciencias Biológicas de la UNAM por el apoyo logístico y al Consejo Nacional de Ciencia y Tecnología (CONACYT) por las becas otorgadas para realizar estudios de posgrado.

\section{RESUMEN}

Introducción: La relación parásito-hospedero entre Dendroctonus y Pinus es reconocida como una amenaza importante para los bosques de coníferas, debido a que puede causar una alta mortalidad de árboles y consecuentemente una pérdida de cobertura forestal.

Objetivo: Identificar potenciales interacciones parásito-huésped entre descortezadores y pinos, así como la contribución de las condiciones ambientales para el establecimiento de estas interacciones ecológicas.

Métodos: Se realizaron modelos de nicho ecológico y análisis de redes ecológicas con la plataforma web de minería de datos espaciales SPECIES (http://species.conabio.gob. $\mathrm{mx} /$ ). Se incluyeron siete especies de descortezadores, 52 de pinos y 19 variables bioclimáticas del portal WorldClim. Resultados: Se identificaron 140 interacciones potenciales, de las cuales el $42 \%$ no han sido registradas previamente. El análisis de redes ecológicas nos permitió caracterizar las relaciones parásito-hospedero en generalistas y especialistas. En cuanto al análisis de nicho 
ecológico, se determinó la distribución potencial de los descortezadores combinando las distribuciones de Pinus y variables climáticas como predictores. Se observó que las variables climáticas contribuyen de forma negativa para la mayoría de las especies de Dendroctonus, es decir, estas variables son restrictivas en casi toda su distribución, por el contrario, las variables bióticas (Pinus) fueron positivas e informativas sobre la mayoría de las distribuciones.

Conclusiones: Los patrones de coexistencia de descortezadores y pinos nos proporcionan información sobre las interacciones que se pueden establecer entre estas especies y que hasta hoy pueden ser desconocidas. Además, la integración de factores bióticos (hospederos) y abióticos (clima), permite obtener modelos de distribución geográfica que caracteriza las regiones con condiciones favorables para la presencia de las especies, así como las especies de hospederos con los que podrían estar interactuando en dichas regiones. Considerando el riesgo fitosanitario por descortezadores, nuestros resultados brindan escenarios geográficos y ecológicos de riesgo donde infestaciones por descortezadores podrían darse, así como la posible emergencia de nuevas interacciones parásito-hospedero desconocidas hasta hoy. Estos modelos son una herramienta que en el futuro pueden dirigir esfuerzos de trabajo de campo para validar y complementar nuestros resultados.

Palabras clave: infestaciones; minería de datos; sanidad forestal; insectos; coníferas.

\section{REFERENCIAS}

Alvarado, D., Bauner, M., \& Galindo, J. (1991). Declinación y muerte del bosque de oyamel (Abies religiosa) en el sur del Valle de México. Agrociencia, Serie Recursos Naturales Renovables, 3(1), 123-143.

Álvarez-Zagoya, R., Pérez, G., Hinojosa-Ontiveros, G. A., \& Salas-Quiñones, J. J. (2019). Prevención de daños contra el barrenador de yemas Rhyacionia sp. en plantaciones de Pinus greggii Engelm., en Nuevo Ideal, Durango, México. Entomología Forestal, 6(1), 471-476.

Armendáriz-Toledano, F., \& Zúñiga, G. (2017). Illustrated key to species of genus Dendroctonus (Coleoptera: Curculionidae) occurring in Mexico and Central America. Journal of Insect Science, 17(2), 1-15.

Armendáriz-Toledano, F., Zúñiga, G., García-Román, J., Valerio-Mendoza, O., \& García-Navarrete, P. G. (2018). Guía ilustrada para identificar a las especies del género Dendroctonus presentes en México y Centroamérica. Red Temática de Salud Forestal; Comisión Nacional de Ciencia y Tecnología; Instituto Politécnico Nacional, México.

Bentz, B., Régnière, J., Fettig, C. J., Hansen, E. M., Hayes, J. L., Hicke, J. A., Kelsey, R. G., Negrón, J. F., \& Seybold, S. J. (2010). Climate change and bark beetles of the western United States and Canada: direct and indirect effects. BioScience, 60(8), 602-613.
Berzunza-Cruz, M., Rodríguez-Moreno, Á., GutiérrezGranados, G., González-Salazar, C., Stephens, C. R., Hidalgo-Mihart, M., Marina, C. F., Rebollar-Téllez, E. A., Bailón-Martínez, D., Balcells, C. D., IbarraCerdeña, C. N., Sánchez-Cordero, V., \& Becker, I. (2015). Leishmania (L.) mexicana infected bats in Mexico: novel potential reservoirs. PLoS Neglected Tropical Diseases, 9(1), 1-15.

Billings, R. F., Clarke, S. R., Espino, V., Cordón, P., Meléndez, B., Campos, J. R., \& Baeza, G. (2004). Gorgojo descortezador e incendios: una combinación devastadora para los pinares de América Central. Unasylva, 217(55), 15-21.

Cano, A., Martínez, O. U., Torres, L. M., \& Sánchez, J. A. (2005). Determinación de zonas con riesgo al ataque del descortezador Dendroctonus adjunctus Blandford en bosques de coníferas del sureste de Coahuila (Informe $n^{\circ} 20$ ). Instituto Nacional de Investigaciones Forestales, Agrícolas y Pecuarias; Secretaría de Agricultura, Ganadería Desarrollo Rural, Pesca y Alimentación, México. http://www.inifapcirne.gob. $\mathrm{mx} /$ Biblioteca/Publicaciones/189.pdf

Chapman, T. B., Veblen, T., \& Schoennagel, T. (2012). Spatiotemporal patterns of mountain pine beetle activity in the southern Rocky Mountains. Ecology, 93(10), 2175-2185.

Cibrián-Tovar, D. (2009). Manual para la identificación de plagas y enfermedades en plantaciones de árboles de navidad. Comisión Nacional Forestal; Comisión Nacional de Ciencia y Tecnología; Universidad Autónoma de Chapingo, México. https://docplayer. es/22396836-Manual-para-la-identificacion-de-plagas-y-enfermedades-en-plantaciones-de-arboles-denavidad.html

Cibrián-Tovar, D., Alvarado-Rosales, D., \& García-Díaz, S. E. (2007). Enfermedades forestales en México. Universidad Autónoma de Chapingo; Comisión Nacional Forestal; Secretaría de Medio Ambiente y Recursos Naturales, México. http://bibliotecasibe. ecosur.mx/sibe/book/000036646

Comisión Nacional para el Conocimiento y Uso de la Biodiversidad (2021). Catálogo de autoridades taxonómicas de especies de flora y fauna con distribución en México. [Base de datos] SNIB-CONABIO, México.

CONABIO. (2020). Sistema Nacional de Información sobre Biodiversidad de México. Registros de ejemplares [Base de datos]. Comisión Nacional para el Conocimiento y Uso de la Biodiversidad, México. https://www.snib.mx/

Creeden, E. P., Hicke, J. A., \& Buotte, P. C. (2014). Climate, weather, and recent mountain pine beetle outbreaks in the western United States. Forest Ecology and Management, 312(1), 239-251.

del-Val, E., \& Sáenz-Romero, C. (2017). Insectos descortezadores (Coleoptera: Curculionidae) y cambio climático: problemática actual y perspectivas en los 
bosques templados. Revista Especializada en Ciencias Químico-Biológicas, 20(2), 53-60.

Elith, J., Graham, C. H., Anderson, R. P., Dudík, M., Ferrier, S., Guisan, A., Hijmnas, R. J., Huettmann, F., Leathwick, J. R., Lehmann, A., Li, J., Lohmann, L. G., Loiselle, B. A., Manion, G., Moritz, C., Nakamura, M., Nakazawa, Y., Overton, J. M., Peterson, A. T., ... Zimmernann, N. E. (2006). Novel methods improve prediction of species' distributions from occurrence data. Ecography, 29(2), 129-151.

Evangelista, P. H., Kumar, S., Stohlgren, T. J., \& Young, N. E. (2011). Assessing forest vulnerability and the potential distribution of pine beetles under current and future climate scenarios in the interior west of the US. Forest Ecology and Management, 262(3), 307-316.

Fairweather, M. L., McMillin, J., Rogers, T., Conklin, D., $\&$ Fitzgibbon, B. (2006). Field guide to insects and diseases of Arizona and New Mexico forests. USDA Forest Service, Southwestern Region.

Gernandt, D. S., López, G. G., Ortiz, S., \& Liston, A. (2005). Phylogeny and classification of Pinus. Taxon, 54(1), 29-42.

González-Salazar, C., Stephens, C. R., \& Marquet, P. A. (2013). Comparing the relative contributions of biotic and abiotic factors as mediators of species' distributions. Ecological Modelling, 248(1), 57-70.

Gymnosperm Database (11 de noviembre, 2020). Pinus contorta [Fichero de datos]. https://www.conifers. org/pi/Pinus_contorta.php

Hernández-Cruz, M. J., \& Muñoz-Viveros, A. L. (2011). Afidofauna de las plantas silvesstres de Cahuacan, Nicolas Romero, edo. de Méxcio (Hemiptera: Aphidoidea). In A. Equihua-Martínez, E. Estrada-Venegas, J. Acuña-Soto, \& M. P. Cháirez-Grijalva (Eds.), Memoria del XV Simposio Nacional de Parasitología Forestal (pp. 120-124). Colegio de Postgraduados, México.

Hicke, J. A., Logan, J. A., Powell, J., \& Ojima, D. S. (2006). Changing temperatures influence suitability for modeled mountain pine beetle (Dendroctonus ponderosae) outbreaks in the western United States. Journal of Geophysical Research, 111(G2), 1-12.

Hijmans, R. J., Cameron, S. E., Parra, J. L., Jones, P. G., \& Jarvis, A. (2005). Very high-resolution interpolated climate surfaces for global land areas. International Journal of Climatology: A Journal of the Royal Meteorological Society, 25(15), 1965-1978.

Jiménez-Martínez, E. (2005). Insectos descortezadores de pino y sus principales depredadores naturales (Informe $n^{\circ} 9$ ). Instituto Nacional Forestal; Universidad Nacional Agraria, Nicaragua. https://repositorio.una. edu.ni/2414/1/nh10j61.pdf

Kelley, S. T., \& Farrell, B. D. (1998). Is specialization a dead end? The phylogeny of host use in
Dendroctonus bark beetles (Scolytidae). Evolution, 52(6), 1731-1743.

Liston, A., Robinson, W. A., Piñero, W. D., \& Álvarez-Buylla, E. R. (1999). Phylogenetics of Pinus (Pinaceae) based on nuclear ribosomal DNA internal transcribed spacer region sequences. Molecular Phylogenetics and Evolution, 11(1), 95-109.

Maldonado, M., Cera, C., Mendoza, A., Sáenz, L., Torres, O., Bravo, P., \& Alatorre, C. (2015). RN 99 Distribución potencial de Dendroctonus valens mediante modelos de máxima entropía: estado de California, E.U. Revista Latinoamericana el Ambiente y las Ciencias, 6(12), 194-198.

Martínez-Méndez, N., Aguirre-Planter, E., Eguiarte, L. E., \& Jaramillo-Correa, J. P. (2016). Modelado de nicho ecológico de las especies del género Abies (Pinaceae) en México: Algunas implicaciones taxonómicas y para la conservación. Botanical Sciences, 94(1), 362-371.

Méndez-Encina, F. M., Méndez-González, J., \& CeranoParedes, J. (2020). Distribución actual y potencial de Dendroctonus mexicanus Hopkins bajo dos escenarios de cambio climático. Madera y Bosques, 26(2), $1-14$.

Mendoza, M. G., Salinas-Moreno, Y., Olivob-Martínez, A., \& Zúñiga, G. (2011b). Factors Influencing the Geographical Distribution of Dendroctonus rhizophagus (Coleoptera: Curculionidae: Scolytinae) in the Sierra Madre Occidental, México. Environmental Ecology, 40(3), 549-559.

Mendoza, M. G., Salinas-Moreno, Y., \& Zúñiga, G. (2011a). Distribución potencial de Dendroctonus rhizophagus Thomas \& Bright (Coleoptera: Curculionidae: Scolytinae) en la Sierra Madre Occidental. In A. Equihua-Martínez, E. Estrada-Venegas, J. AcuñaSoto, \& M. P. Cháirez-Grijalva (Eds.), Memoria del XV Simposio Nacional de Parasitología Forestal (pp. 120-124). Colegio de Postgraduados, México.

Morales-Rangel, A., Cambrón-Sandoval, V. H., Vergara-Pineda, S., \& Obregón-Zúñiga, J. A. (2016). Fluctuación poblacional de Dendroctonus frontalis Zimmerman, 1868 y Dendroctonus mexicanus Hopkins, 1909 (Coleoptera: Curculionidae: Scolytinae) y su asociación con variables climáticas en bosques de pino en el municipio de Landa de Matamoros, Querétaro, México. Entomología Mexicana, 3(1), 633-638.

Morales-Rangel, A., Cambrón-Sandoval, V. H., SotoCorrea, J. C., Jones, R. W., \& Obregón-Zúñiga, J. A. (2018). Efecto de la temperatura en poblaciones de Dendroctonus frontalis Zimmerman y Dendroctonus mexicanus Hopkins (Coleoptera: Curculionidae: Scolytinae) bajo un escenario de cambio climático en la Sierra Gorda Queretana. Acta Zoológica Mexicana (nueva serie), 34(3), 1-8.

Moreno-Rico, O., Sánchez-Martínez, G., MarmolejoMonsiváis, J. G., Pérez-Hernández, K., \& Moreno-Manzano, C. E. (2015). Diversidad de hongos 
Ophiostomatoides en pinos de la Sierra Fría de Aguascalientes, méxico, asociados con Dendroctonus mexicanus. Revista Mexicana de Biodiversidad, $86(1), 1-8$.

Naturalista (11 de noviembre, 2020). Pinus lawsonii [Fichero de datos]. https://www.naturalista.mx/ taxa/135762-Pinus-lawsonii

Oksanen, J., Blanchet, F. G., Friendly, M., Kindt, R., Legendre, P., McGlinn, D., Minchin, P. R., O`Hara, R. B., Simpson, G. L., Solymos, P., Stevens, M. H., Szoecs, E., \& Wagner, H. (2020). Vegan: Community Ecology Package. (R Package). https://github.com/ vegandevs/vegan

Pietrukiewicz, P., \& Eskiviski, E. (2010). Control biológico del pulgón del pino Cinara atlántica Wilson por el parasitoide Xenostigmus bifasciatus en una plantación de Pinus taeda. (Informe $\mathrm{n}^{\circ}$ 63). Facultad de Ciencias Forestales, Universidad Nacional de Misiones, Argentina. https://inta.gob.ar/sites/default/files/ script-tmp-inta-control-pulgon-pino.pdf

Ramírez-Herrera, C., Vargas-Hernández, J. J., \& LópezUpton, J. (2005). Distribución y conservación de las poblaciones naturales de Pinus greggii. Acta Botánica Mexicana, 72(1), 1-16.

Régnière, J., \& Bentz, B. (2007). Modeling cold tolerance in the mountain pine beetle, Dendroctonus ponderosae. Journal of Insect Physiology, 53(6), 559-572.

Rivera-Rojas, M., Locatelli, B., \& Billings, R. (2010). Cambio Climático y eventos epidémicos del gorgojo descortezador del pino Dendroctonus frontalis en Honduras. Forest Systems 19(1) 70-76.

Ruíz-Cancino, E., Coronado, J. M., \& Escamilla, O. S. (1997). Insectos plaga de Pinus cembroides Zucc. y $P$. nelsoni Shaw en el suroeste de Tamaulipas, México. BioTam, 8(2/3), 41-48.

Salinas-Moreno, Y., Mendoza, M. G., Barrios, M. A., Cisneros, R., Macías-Sámano, J., \& Zúñiga, G. (2004). Areography of the genus Dendroctonus (Coleoptera: Curculionidae: Scolytinae) in Mexico. Journal of Biogeography, 31(7), 1163-1177.

Salinas-Moreno, Y., Vargas, C. F., Zúñiga, G., Victor, J., Ager, A., \& Hayes, J. L. (2010). Atlas de distribución geográfica de los descortezadores del género Dendroctonus (Curculionidae: Scoliytinae) en México/ Atlas of the geographic distribution of bark beetles of the genus Dendroctonus (Curculionidae: Scoliytinae) in Mexico. Instituto Politécnico Nacional; Comisión Nacional Forestal, México.

Sánchez-González, A. (2008). Una visión actual de la diversidad y distribución de los pinos de México. Madera y Bosques, 14(1), 107-120.

Santana-Zarza, D. M., Barraza-Domínguez, J. E., \& Castillo-Jiménez, I. (2020). Distribución potencial del género Dendroctonus (Coleoptera: Curculionidae) en el municipio de Amanalco, estado de México. Entomología Mexicana, 7(1), 348-354.

Sierra, R., \& Stephens, C. R. (2012). Exploratory analysis of the interrelations between co-located boolean spatial features using network graphs. International Journal of Geographical Information Science, 26(3), 441-468.

Six, D. L., \& Bracewell, R. (2015). Dendroctonus. In F. E. Vega, \& R. W. Hofstetter (Eds.), Bark Beetles biology and ecology of native and invasive species (Chapter 8, pp. 305-350). Academic Press.

Sosa, L., Méndez, J., García, M. A., Cambrón-Sandoval, V. H., Villarreal, J. A., Ruíz, C. G., \& Montoya, J. C. (2018). Distribución potencial de barrenadores, defoliadores, descortezadores y muérdagos den bosques de coníferas de México. Revista Mexicana de Ciencias Forestales, 9(47), 187-208.

Soto-Correa, J. C., Avilés-Carrillo, I., Girón-Gutiérrez, D., \& Cambrón-Sandoval, V. H. (2018). Abundancia altitudinal de Dendroctonus frontalis (Coleoptera: Curculionidae) en relación a variables climáticas en Hidalgo, México. Revista de Biología Tropical, 67(3), 370-379.

Soto-Correa, J. C., Girón-Gutiérrez, D., \& CambriónSandoval, V. H. (2020). Coloración y abundancia de Dendroctonus mexicanus Hopkins, 1905 en cuatro regiones de México. Revista Mexicana de Ciencias Forestales, 11(59), 163-184.

Stephens, C. R., González-Salazar, C., Sánchez-Cordero, V., Becker, I., Rebollar-Tellez, E., Rodríguez-Moreno, Á., Berzunza-Cruz, M., Balcells, C., GutiérrezGranados, G., Hidalgo-Mihart, M., Ibarra-Cedeña, C. N., Ibarra, M. P., Iñiguez, L. I., \& Ramírez, M. M. (2016). Can you judge a disease host by the company it keeps? Predicting disease hosts and their relative importance: a case study for Leishmaniasis. PLoS Neglected Tropical Diseases, 10(10), e0005004.

Stephens, C. R., Heau, J., González, C., Ibarra-Cerdeña, C. N., Sánchez-Cordero, V., \& González-Salazar, C. (2009). Using biotic interaction networks for prediction in biodiversity and emerging diseases. PLoS One, 4(5), e5725.

Stephens, C. R., Sierra-Alcocer, R., González-Salazar, C., Barrios, J. M., Salazar, J. C., Robredo, E., \& del Callejo, E. (2019). SPECIES: A platform for the exploration of ecological data. Ecology and Evolution, 9(4), 1638-1653.

Ungerer, M. J., Ayres, M. P., \& Lombardero, M. J. (1999). Climate and the northern distribution limits of Dendroctonus frontalis Zimmermann (Coleoptera: Scolytidae). Journal of Biogeogeraphy, 26(6), 1133-1145

Víctor, J., \& Zúñiga, G. (2016). Phylogeny of Dendroctonus bark beetles (Coleoptera: Curculionidae: Scolytinae) inferred from morphological and molecular data. Systematic Entomology, 41(1), 162-177. 
Weed, A., Bentz, B., Ayres, M. P., \& Holmes, T. P. (2015). Geographically variable response of Dendroctonus ponderosae to winter warming in the western United States. Landscape Ecology, 30(6), 1075-1093.

Wood, S. L. (1963). A revision of the bark beetle genus Dendroctonus Erichson (Coleoptera: Scolytidae). The Great Basin Naturalist, 23(1/2), 1-117.

World Resources Institute. (1995). World Resources, 1994 95: A Report. Oxford University Press.
Zúñiga, G., Cisneros, R., Hayes, J. L., \& Macías-Sámano, J. (2002). Karyology, Geographic Distribution, and Origin of the Genus Dendroctonus Erichson (Coleoptera: Scolytidae). Annals of the Entomological Society of America, 95(3), 267-275.

Zúñiga, G., Mendoza, M. G., Cisneros, R., \& SalinasMoreno, Y. (1999). Zonas de sobreposición en las áreas de distribución geográfica de las especies mexicanas de Dendroctonus Erichson (Coleoptera: Scolytidae) y sus implicaciones ecológico-evolutivas. Acta Zoológica Mexicana (nueva serie), 77(1), 1-22. 